\title{
Virulence factor landscape of a Staphylococcus aureus sequence type 45 strain, MCRF184
}

\author{
Vijay Aswani ${ }^{1 \dagger}$, Fares Najar ${ }^{2 \dagger}$, Madhulatha Pantrangi ${ }^{3}$, Bob Mau ${ }^{4}$, William R. Schwan ${ }^{5}$ and Sanjay K. Shukla ${ }^{3 *}$
}

\begin{abstract}
Background: We describe the virulence factors of a methicillin-sensitive Staphylococcus aureus sequence type (ST) 45 strain, MCRF184, (spa type t917), that caused severe necrotizing fasciitis in a 72-year-old diabetic male. The genome of MCRF184 possesses three genomic islands: a relatively large type III vSaa with 42 open reading frames (ORFs) that includes superantigen- and lipoprotein-like genes, a truncated $v S a \beta$ that consists mostly of the enterotoxin gene cluster (egc), and a vSay island with $18 \mathrm{ORFs}$ including a-toxin. Additionally, the genome has two phage-related regions: phage $\varphi S a 3$ with three genes of the immune evasion cluster (IEC), and an incomplete phage that is distinct from other $S$. aureus phages. Finally, the region between orfX and orfY harbors a putative efflux pump, acetyltransferase, regulators, and mobilization genes instead of genes of SCCmec.

Results: Virulence factors included phenol soluble modulins (PSMs) a1 through a4 and PSMs $\beta 1$ and $\beta 2$. Ten ORFs identified in MCRF184 had not been reported in previously sequenced S. aureus strains.

Conclusion: The dire clinical outcome in the patient and the described virulence factors all suggest that MCRF184, a ST45 strain is a highly virulent strain of S. aureus.
\end{abstract}

Keywords: Staphylococcus aureus, Virulence factors, Necrotizing fasciitis, ST45, enterotoxin gene cluster

\section{Background}

The ability of $S$. aureus to colonize and infect humans comes from a large arsenal of virulence genes including genes for proteins to attach to host tissue, tissue-degrading enzymes, leukocidins, antibiotic-resistance, pyrogenic toxins, and immunomodulating proteins [1]. A number of $S$. aureus genomes have been sequenced to identify potential new virulence genes or novel combinations of known virulence genes [2]. These studies have led to the identification of new genomic islands and genetic elements, which harbor known and putative toxins, phenol-soluble modulins, and accessory genes to virulence [3-6]. Differences in virulence of $S$. aureus strains, however, may be due to even small differences in genome sequence: Kennedy et al [7] studied genetic variation in USA300 MRSA strains and found that large differences in virulence in a mouse sepsis model occurred among strains with relatively few genetic differences. Single SNP differences have recently been demonstrated to underpin the virulence of some strains $[8,9]$. Similarly, the insertion of IS256 (a transposable element) into the promotor of the rot gene increased virulence [10]. Panton-Valentine leukocidin (PVL), a major virulence factor of $S$. aureus has been shown to have a direct role in necrotizing fasciitis [5]. We describe here the virulence traits of MCRF184, a methicillin-sensitive, ST45 strain that caused a debilitating necrotizing fasciitis in a diabetic man, necessitating the amputation of the patient's leg to save his life.

\footnotetext{
* Correspondence: shukla.sanjay@marshfieldresearch.org

${ }^{\dagger}$ Vijay Aswani and Fares Najar contributed equally to this work.

${ }^{3}$ Center for Human Genetics, 1000 North Oak Avenue \# MLR, Marshfield, WI

54449, USA

Full list of author information is available at the end of the article
}

(c) The Author(s). 2019 Open Access This article is distributed under the terms of the Creative Commons Attribution 4.0 International License (http://creativecommons.org/licenses/by/4.0/), which permits unrestricted use, distribution, and 


\section{Results}

Overview of antimicrobial resistance and virulence gene content

MCRF184 is a methicillin-susceptible strain that belongs to sequence type (ST) 45 and spa type t917. This strain was recovered during both the early and late stages of the infection of the leg [11]. Among some of the known virulence factors of S. aureus, the genome of this strain harbored clumping factors genes clfA and $c l f B$, fibronectin binding protein gene $f n b A$ but not $f n b B$, collagen binding adhesion gene cna, intracellular adhesion gene icaA and newly identified toxin genes $b s a$, staphylococcal superantigen-like gene 1 (ssl1), and lpl110 (Table 1). The staphylococcal enterotoxins, staphylococcal superantigen-like $(s s l)$ genes, and genes involved in immune evasion were present on mobile genetic elements. MCRF184 was negative for toxic shock syndrome toxin (tst), and the Panton-Valentine leucocidin (lukSF-PV).

\section{Mobile genetic elements}

The MCRF184 strain harbored six MGEs (Fig. 1): $\nu \mathrm{Sa} \alpha, \nu \mathrm{Sa} \beta, v \mathrm{Sa} \gamma, \phi \mathrm{Sa} 3$, an incomplete phage, and a newly identified region between orf $X$ and $\operatorname{orf} Y$, named $\mathrm{MGE}^{X Y}$ that also harbored mobilization genes. The incomplete phage has not been previously described, and the $\mathrm{MGE}^{X Y}$ harbored novel combinations of sequences. The MCRF184 genome did not include intact pathogenicity islands [12], plasmids, or integrative conjugative elements (ICE6013, Tn916/Tn5801) [13].

\section{Genomic islands of MCRF184 \\ vSaa}

Genomic islands, generally 10 to $200 \mathrm{~kb}$ long, are a cluster of genes acquired by horizontal transfer [14]. The $v \mathrm{Sa} \alpha$ was a type III genomic island (Fig. 2) and harbored alleles of eight ssl and seven lipoprotein-like $(l p l)$ genes. The $v S a \alpha$ region was nearly identical to vSa $\alpha$ of two other ST45 strains, CA-347 [15] and an unpublished genome, CFSAN007835 (GenBank \# CP017685.1). In $v$ Sa $\alpha$, eleven SNPs accounted for the differences between MCRF184 and CA347 (Table 2), eight of which were in protein coding regions-five of which would result in amino acid substitutions and one in a truncated protein in both MCRF184 and CA347. All but two of these changes in coding regions were in hypothetical proteins; of the two other changes, one was in an exotoxin gene and the other in the host specificity gene, hsdS (CKU_0369) of the restriction modification system.

\section{$v S a \beta$}

The $\nu \mathrm{Sa} \beta$ of MCRF184 was truncated compared to $v \mathrm{Sa} \beta$ in MW2 and USA300FPR3757. It harbored eleven ORFs including the enterotoxin gene cluster (egc) genes: seg, sen, seu sei, sem and seo (Fig. 3), and was nearly identical in genes present in all three ST45 strains. Four genetic differences were noted in the $v \mathrm{Sa} \beta$ islands between MCRF184 and CA347 strain (Table 3), three of which were single nucleotide polymorphisms (SNPs). A significant additional difference was the deletion of two transposases in MCRF184, but present in CA347 strain. Furthermore, one of the SNPs in sen would lead to a truncated protein in MCRF184. The region containing two genes - a rep gene coding for a helicase and a second gene coding for a hypothetical protein, between positions 1,785,972 and 1787, 688 were unique to the three CC45 strains and not found in other $S$. aureus $\nu \mathrm{Sa} \beta$ islands. The observation that a hypothetical protein and the helicase were found in the three ST45 strains but absent from the other $v \mathrm{Sa} \beta$ islands sequenced could be of significance for the ST45 strains' pathogenicity.

The genomic islands, $\nu \mathrm{Sa} \alpha$ and $\nu \mathrm{Sa} \beta$ generally exist in four allelic forms in $S$. aureus strains and their specificity is determined by the structural differences in $h s d S$ (host specificity determinant), a rapidly evolving gene with amino acid sequence level identity across the S. aureus genomes of less than 66\% [3]. $\nu \mathrm{Sa} \beta$ lacked the hsdS (Fig. 3).

\section{vSay}

Comparison of the $v$ Say sequence between the two other ST45 strains, MCRF184 and CA-347, revealed conserved gene order and no amino acid differences. Comparing nucleotide and amino acid sequences between them (Table 4), there were only three differences in protein-coding regions, none of which resulted in an amino acid change. A comparison with other ST types S. aureus strains showed conservation of gene composition.

This genomic island contains the IEC2 cluster, including the $\alpha$-haemolysin (Hla) and the prototype $\beta$ PFT of S. aureus. The $\nu$ Say (Fig. 4) was flanked by the genes murI (glutamic racemase) and argF (ornithine transcarbamoylase subunit F). It additionally contained three more $s s l s$.

\section{The phages of MCRF184 $\varphi \mathrm{Sa} 3$}

The $\phi S a 3$ (Fig. 5) was inserted into the $h l b$ gene, making it a $\beta$-hemolysin-converting bacteriophage $(\beta C-\phi)$. This phage is known to carry IEC1, which is variable in gene content among strains [16]. In MCRF184, IEC1 consists of sak - (truncated amidase) - chp - scn suggesting that it is an IEC type B [17]. The truncated amidase is not 
Table 1 Major virulence-related genes in S. aureus strain, MCRF184

\begin{tabular}{|c|c|c|}
\hline & Locus & Location \\
\hline \multirow[t]{7}{*}{ Enterotoxins } & CKU_1443 SE & core \\
\hline & CKU_1636 seg & $v S a \beta$ \\
\hline & CKU_1637 sen & $\checkmark \mathrm{Sa} \beta$ \\
\hline & CKU_1638 seu & $v S a \beta$ \\
\hline & CKU_1639 sei & $v S a \beta$ \\
\hline & CKU_1640 sem & $v S a \beta$ \\
\hline & CKU_1641 seo & $v S a \beta$ \\
\hline \multirow[t]{11}{*}{ Exotoxins } & CKU_0360 ss/1 & vSaa \\
\hline & CKU_0361 ss/2 & vSaa \\
\hline & CKU_0362 ss/4 & vSaa \\
\hline & CKU_0363 ss/3 & vSaa \\
\hline & CKU_0365 ss/5 & vaa \\
\hline & CKU_0366 ss/9 & vSaa \\
\hline & CKU_0367 ss/10 & vsaa \\
\hline & CKU_0370 ss/11 & vaa \\
\hline & CKU_0998 ss/12 & vay \\
\hline & CKU_0999 ss/13 & vay \\
\hline & CKU_1000 ss/14 & vay \\
\hline Exfoliative toxin & CKU_1005 eta & vSay \\
\hline Alpha-hemolysin & CKU_0995 hla & vay \\
\hline Beta-hemolysin & CKU_1753 h/b & $\phi \mathrm{Sa3}$ \\
\hline Delta-hemolysin (RNAllI) & CKU_2494 hld & core \\
\hline Gamma-hemolysin Component & CKU_2175 hlgA & core \\
\hline Gamma-hemolysin Component & CKU_2176 hlgC & core \\
\hline Gamma-hemolysin Component & CKU_2177 hlgB & core \\
\hline \multicolumn{3}{|l|}{ Adhesins } \\
\hline Collagen-binding protein & CKU_2442 cna & core \\
\hline Fibronectin-adhesin & CKU_2253 fnbA & core \\
\hline Elastin adhesin & CKU_1327 ebpS & core \\
\hline Laminin-adhesin & CKU_0713 eno & core \\
\hline Fibrinogen & CKU_0723 ClfA & core \\
\hline Fibrinogen & CKU_2384 ClfB & core \\
\hline Fibrinogen & CKU_0989 fib & core \\
\hline Fibrinogen & CKU_0496 sdrC & core \\
\hline \multicolumn{3}{|l|}{ Exoenzymes } \\
\hline Serine protease & CKU_0857 htrA & core \\
\hline Serine V8 protease & CKU_0881 sspA & core \\
\hline Cysteine protease & CKU_0880 sspB & core \\
\hline Cysteine protease & CKU_0879 sspC & core \\
\hline Lipase precursor & CKU_0273 geh & core \\
\hline Lipase precursor & CKU_2426 gehC & core \\
\hline Lipase & CKU_0588 lipA & core \\
\hline Esterase & CKU_2106 & core \\
\hline Hyaluronate lyase & CKU_1961 hysA2 & core \\
\hline Termonuclease & CKU_1173 nucH & core \\
\hline Cell wall hydrolase & CKU_1081 lytN & core \\
\hline Zinc metalloprotease & CKU_1096 & core \\
\hline Clp protease proteolytic subunit & CKU_0704 clpP & core \\
\hline
\end{tabular}

Table 1 Major virulence-related genes in S. aureus strain, MCRF184 (Continued)

\begin{tabular}{|c|c|c|}
\hline & Locus & Location \\
\hline Clp protease ATP binding subunit & CKU_0813 clpB & core \\
\hline Clp protease ATP binding subunit & CKU_1509 clpX & core \\
\hline Clp protease ATP binding subunit & CKU_2300 clpL & core \\
\hline \multicolumn{3}{|l|}{ Phenol Soluble Modulins } \\
\hline PSMa1 & 426,966 to 426,902 & core \\
\hline PSMa2 & 426,870 to 426,805 & core \\
\hline PSMa3 & 426,752 to 426,685 & core \\
\hline PSMa4 & 426,620 to 426,559 & core \\
\hline PSM 1 & CKU_1007 & core \\
\hline PSM $\beta 2$ & CKU_1008 & core \\
\hline PSM $\delta$ & $1,955,755$ to $1,955,676$ & core \\
\hline \multicolumn{3}{|l|}{ Immunomodulators } \\
\hline Staphylokinase & CKU_1760 sak & $\phi \mathrm{Sa} 3$ \\
\hline Chemotaxis inhibiting protein & CKU_1758 chp & $\phi \mathrm{Sa} 3$ \\
\hline Complement inhibitor & CKU_1757 & $\phi \mathrm{Sa3}$ \\
\hline Immunoglobulin $\mathrm{G}$ binding protein $\mathrm{A}$ & CKU_0065 spa & core \\
\hline Immunoglobulin $\mathrm{G}$ binding protein & CKU_2174 sbi & core \\
\hline Lipoprotein like gene products & CKU_2474 Ip/1 & vSaa \\
\hline Lipoprotein like gene products & CKU_0373 Ip/2 & $v$ Saa \\
\hline Lipoprotein like gene products & CKU_0374 lp/3 & $v$ Saa \\
\hline Lipoprotein like gene products & CKU_0375 Ip/4 & $v$ Saa \\
\hline Lipoprotein like gene products & CKU_0376 /p/5 & $v$ Saa \\
\hline Lipoprotein like gene products & CKU_0377 Ip/6 & vaa \\
\hline Lipoprotein like gene products & CKU_0378 lp/ & vSaa \\
\hline \multicolumn{3}{|l|}{ Virulence related genes } \\
\hline \multirow[t]{5}{*}{ Biofilm genes } & CKU_2420 icaR & core \\
\hline & CKU_2421 icaA & core \\
\hline & CKU_2422 icaD & core \\
\hline & CKU_2423 icaB & core \\
\hline & CKU_2424 icaC & core \\
\hline Leukocidin GH & CKU_1786 lukGH & core \\
\hline \multicolumn{3}{|l|}{ Regulatory genes } \\
\hline \multirow[t]{2}{*}{ S. aureus exotoxin (SaeRS) } & CKU_0640 saes & core \\
\hline & CKU_0641 saeR & core \\
\hline Staphylococcal accessory regulator (sarA) & CKU_0551 sarA & core \\
\hline Sigma factor B & CKU_1825 sigB & core \\
\hline Repressor of Toxins & CKU_1594 rot & core \\
\hline
\end{tabular}

unique to MCRF184, and an intact amidase is upstream of sak and forms part of the endolysin-holin lytic module of the phage. There was also lukGH genes located downstream of the phage element (Fig. 5), representing a core genome virulence factor in MCRF184.

\section{An incomplete phage}

A novel incomplete phage was located between nucleotide positions $1,242,209$ to $1,258,118$ (Fig. 6). 


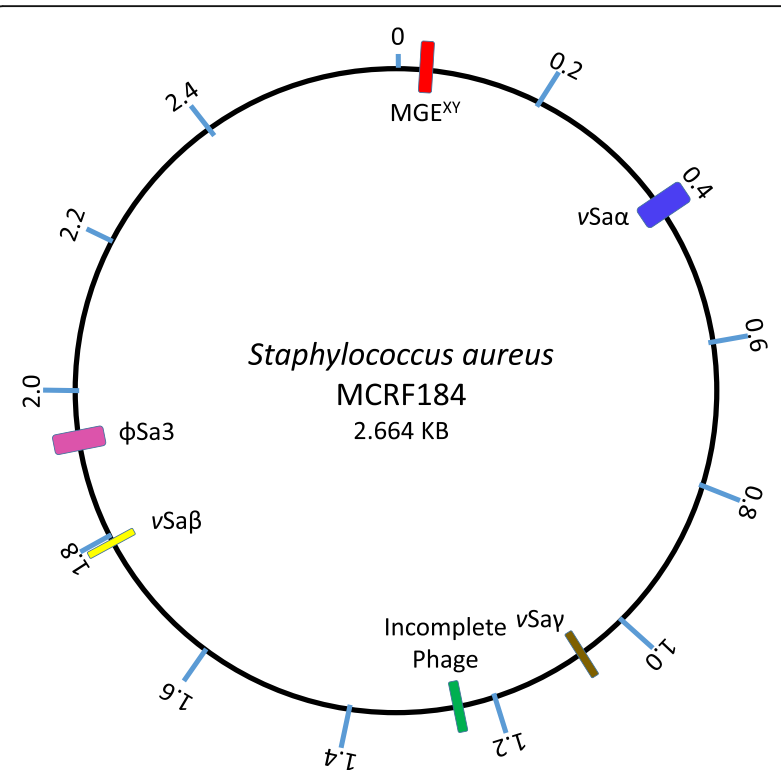

Fig. 1 Circular representation of the MCRF184 genome. Virulence genomic islands are marked: vSaa [blue], vSa [yellow], vSay [brown], Incomplete phage [green], $\varphi S a 3$ [pink] and $\mathrm{MG}^{\mathrm{XY}}$ [red]

PHASTER analysis found it to be an incomplete prophage (PHASTER score $40 ;<70$ considered incomplete). Twenty of the 27 proteins were identified as phage proteins. Three of the 27 proteins matched staphylococcal phage $\phi$ NM3. The complete sequence of this incomplete phage had a $>99 \%$ sequence identity with ST45 strains CA-347 and CFSAN007835. The gene content was unusual in having a terminase large subunit gene (ter $L)$ instead of a small subunit gene (ter $S$ ), and in having a phage head morphogenesis gene. Interestingly, SaPIbov5 is known to have terL but not terS, and is mobilized by both pac- and cos-type helper phages [16]. The glutamine synthetase gene is not known to be used as an integration site by SaPIs, but it is used by an unrelated $30 \mathrm{~kb}$ phage, $\phi 909$ described in S. epidermidis [18]. The integrase of this incomplete phage was distinct from the groups defined for S. aureus phage [19] and SaPIs [20].

\section{$M G E^{X Y}$}

The SCCmec cassette in MRSA is usually present at the 3' end of the conserved gene $\operatorname{orf} X$, an rRNA methyltransferase at a position $\sim 34,000$ base pairs from the origin of the replication [21]. The region between $\operatorname{orf} X$ and $\operatorname{orf} Y$, a tRNA dihydrouridine synthetase is known to be highly variable in gene content among S. aureus strains [22, 23]. In MCRF184, this region has a series of restriction-modification genes ( $h s d R, h s d M$, and R-M type III) and a unique combination of putative antimicrobial resistance genes (emrB/qacA, tetR) located near the mobilization genes, int and tnp for transposon and integrase (Fig. 7). The putative efflux pump, emrB/qacA, is among those known for $S$. aureus [24]. The position of the $h s d R$ and $h s d M$ genes and the R-M type III system in this location of the $S$. aureus genome appears to be well conserved (Fig. 7). However, the presence of $e m r B / q a c A$, tetR and int and tnp in this region appear unique to MCRF184, CA-347, CFSAN007835 (all ST45 types) and an ST508 S. aureus isolated from a Buruli ulcer [25].

\section{The phenol soluble modulins of MCRF184}

Phenol soluble modulins (PSMs) are a family of amphipathic, alpha-helical peptides that have multiple roles in pathogenesis and are critical determinants of staphylococcal virulence [26]. In MCRF184, we identified all four $\alpha$ peptides, the two $\beta$ peptides and the $\delta$-peptide (Additional file 1: Figure S1A and B). We further confirmed the PSMs by determining their predicted structures: characteristic $\alpha$-helical secondary structures that were amphipathic-hydrophilic on one side and hydrophobic on the other-using PEP-FOLD $[27,28]$ for the alpha PSMs and SWISS-MODEL Workspace [29] for the beta PSMs (Additional file 1: Figure S1C).

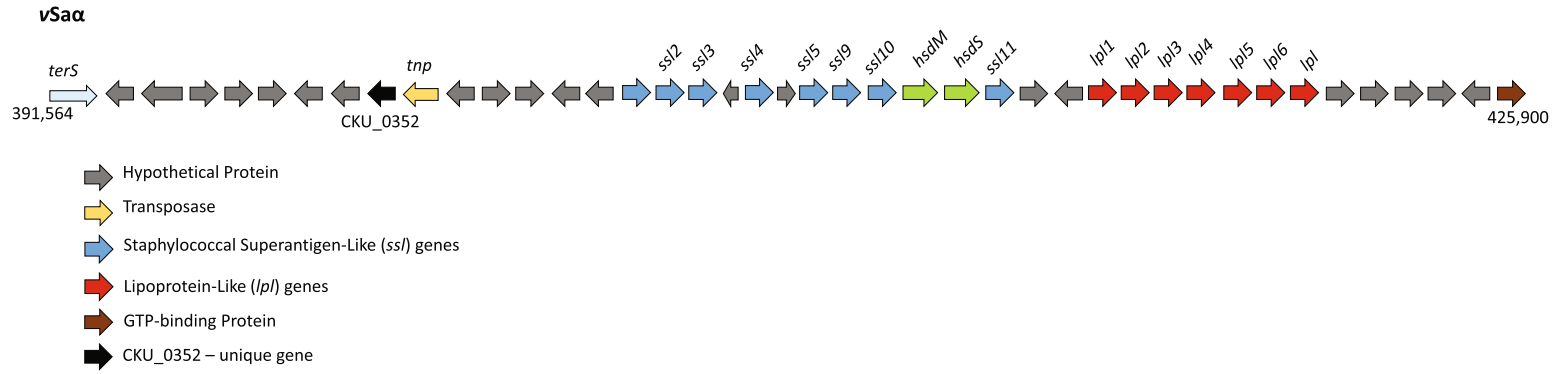

Fig. 2 vSaa genomic island showing lipoprotein-like and staphylococcal superantigen like genes along with mobile genetic elements 
Table 2 Comparison of vSaa of MCRF184 with the strain, CA347 identifying SNP differences between them

\begin{tabular}{|c|c|c|c|c|c|c|c|c|}
\hline Nucleotide position & AA Change & Region & CDS & CDS Position & Change & Codon Change & Polymorphism Type & Protein Effect \\
\hline 392,566 & $A->T$ & $\mathrm{HP}$ & CKU_2476 & 292 & $C->T$ & $\mathrm{GCT}->\mathrm{ACT}$ & SNP (transition) & Substitution \\
\hline 395,313 & & non-coding & & & $A->C$ & & SNP (transversion) & $n / a$ \\
\hline 398,723 & & $\mathrm{HP}$ & CKU_0352 & 161 & $A->T$ & & SNP (transversion) & Truncation \\
\hline 399,587 & $A->T$ & $\mathrm{HP}$ & CKU_0355 & 52 & $C->T$ & GCA - $>$ ACA & SNP (transition) & Substitution \\
\hline 401,239 & & non-coding & & & $T->C$ & & SNP (transition) & $\mathrm{n} / \mathrm{a}$ \\
\hline 401,244 & & non-coding & & & $A->G$ & & SNP (transition) & $\mathrm{n} / \mathrm{a}$ \\
\hline 404,499 & $1->K$ & exotoxin & CKU_0361 & 473 & $\mathrm{~T}->\mathrm{A}$ & ATA $->$ AAA & SNP (transversion) & Substitution \\
\hline 412,382 & $1->V$ & hsdS & CKU_0369 & 76 & $A->G$ & ATT - > GTT & SNP (transition) & Substitution \\
\hline 418,429 & $G->W$ & $\mathrm{HP}$ & CKU_0374 & 658 & $G->T$ & GGG - > TGG & SNP (transversion) & Substitution \\
\hline 423,848 & & $\mathrm{HP}$ & CKU_0381 & 168 & $C->T$ & GGC - > GGT & SNP (transition) & None \\
\hline 423,933 & $F->V$ & $\mathrm{HP}$ & CKU_0381 & 253 & $\mathrm{~T}->\mathrm{G}$ & $\Pi \pi \mathrm{T}->\mathrm{GTT}$ & SNP (transversion) & Substitution \\
\hline
\end{tabular}

\section{Discussion}

The whole genome sequence analysis of MCRF184, a clinically virulent and aggressive strain showed virulence features in common with two other ST45 strains, CA-347 and CFSAN007835 available in GenBank. However, these virulence features of the other genomes have not been described. Our analysis of the genomic islands of MCRF184 points to several distinctive virulence features: a streamlined $v \mathrm{Sa} \beta$ that mostly consists of the $e g c$, and an $\mathrm{MGE}^{\mathrm{XY}}$ that appears to be unique to ST45 strains of $S$. aureus.

With regards to the virulence factors of the $v S a \alpha$, Nguyen [30] showed that deletion of the lpl cluster, which is also present in the $v \mathrm{Sa \alpha}$ genomic island of MCRF184, prevents the stimulation of the production of proinflammatory cytokines in human monocytes, macrophages, and keratinocytes. They further demonstrated that purified lipoprotein, Lpl1 was able to elicit a TLR2-dependent response and that heterologous expression enhanced their immune stimulatory activity, particularly contributing to the invasion of $S$. aureus into human keratinocytes and mouse skin, compared to cells without these virulence genes. Thus, the lpl cluster of MCRF184 $v \mathrm{Sa} \alpha$ may help stimulate virulence by stimulating a host inflammatory response that can cause symptoms of pain, swelling, erythema and fever.

The egc in MCRF184 encodes six genes, which belong to a superantigen family that are capable of triggering a massive toxic shock response [31]. Proteins encoded by $e g c$ are not reported to be highly immunogenic, but they can evade immune response due to lack of neutralization by the human sera [32]. In a comprehensive study done by Roetzer et al [33], it has been shown that 1) supernatants from a strain harboring egc were sufficient for a lethal outcome in

$v S a \beta$

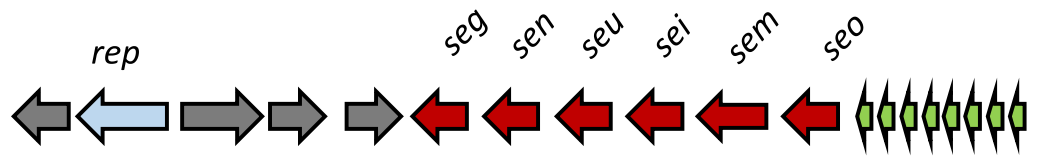

$1,796,739$

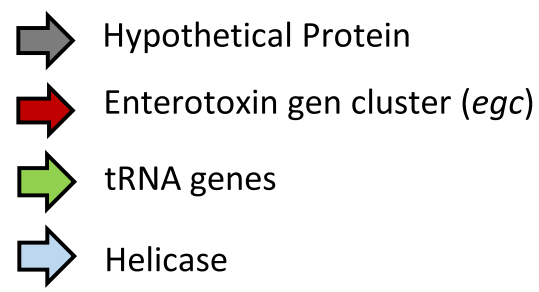

Fig. $3 v S a \beta$ genomic island showing genes of the enterotoxin gene cluster (egc) 
Table 3 Comparison of vSa $\beta$ of MCRF184 with strain, CA347 identifying SNP differences between them

\begin{tabular}{|c|c|c|c|c|c|c|c|c|}
\hline Nucleotide Position & AA Change & Region & CDS & CDS Position & Change & $\begin{array}{l}\text { Codon } \\
\text { Change }\end{array}$ & Polymorphism Type & Protein Effect \\
\hline 1787,882 & & CDS & & & 1223 bases & $\mathrm{n} / \mathrm{a}$ & deletion & $\begin{array}{l}\text { loss of two transposases, } \\
\text { CA347_RS09315 and } \\
\text { CA347_RS09320 }\end{array}$ \\
\hline $1,789,959$ & & noncoding & & & $C->T$ & & SNP (transition) & \\
\hline $1,791,116$ & $\mathrm{M}->\mathrm{STOP}$ & sen & CKU_1637 & 756 & $\mathrm{~T}->\mathrm{A}$ & & SNP (transversion) & truncation \\
\hline $1,795,127$ & $N->D$ & seo & CKU_1641 & 235 & $\mathrm{~T}->\mathrm{C}$ & AAT - > GAT & SNP (transition) & Substitution \\
\hline
\end{tabular}

rabbits, 2) different quantities of egc encoded enterotoxins are produced by S. aureus isolates, 3) 10 nanograms of expressed and purified recombinant SEI and SEN was lethal at $24 \mathrm{~h}$ and $48 \mathrm{~h}$, and 4 ) sei and sen appear to play a more important role in virulence compared to the other egc genes. Stach et al [34], in a rabbit model of infective endocarditis, investigated the role of $t s t H$ and individual genes of $e g c$ and in a USA200 genetic background and noted that proteins from both genes independently contributed to development of vegetation and infective endocarditis. Proteins made by sem, seo, and seu contributed to the vegetation formation, and deletions of $t s t H$ and $e g c$ decreased the vegetation size. Furthermore, Johler et al [35] reported outbreaks of staphylococcal food poisoning and emetic activity from egc-harboring $S$. aureus belonging to clonal complex CC9 and CC45. These observations suggest that even though MCRF184 had a truncated $\nu \mathrm{Sa} \beta$ island, the virulence imparted by the $e g c$ genes alone could account for significant virulence through their modulation of the immune system, particularly in the 72-year-old diabetic male with the life and limb-threating necrotizing fasciitis. Furthermore, the presence of an IEC in $\phi \mathrm{Sa} 3$ could have contributed to evading phagocytosis of the pathogen. Another interesting aspect of the MCRF184 genome is that it had three ferrichrome-binding proteins-fhuA, fhuB, and fhuD-important for growth under iron-restricted conditions [25]. The MCRF184 $\phi S a 3$ was integrated into the $h l b$ and extended to position 2 genes upstream of groEL.

The $\mathrm{MGE}^{X Y}$ region of MCRF184 was identical to the ones found in CA-347 and the $S$. aureus Buruli ulcer isolate [25]. The region encodes a restriction- modification system ( $h s d R / h s d M$ ) and included an ermB/qacA drug resistance transporter gene of the major facilitator superfamily (MFS) including an integrase, a transposase, a tetR/acR family transcriptional regular [36], and a flanking tRNA. The presence of all three types of PSMs-four $\alpha$-types, two $\beta$-types and one $\delta$-type-and their ability to enhance virulence through cytolysis of cells of the immune system and biofilm formation suggest further mechanisms for the enhanced virulence of MCRF184.

Wang et al [6] showed that psm $\alpha$ mutants were severely attenuated in their ability to cause subcutaneous abscesses in the skin of mice compared with the wild-type strain. Thus, the psm $\alpha$ toxins in MCRF184 could have contributed significantly to their virulence in causing necrotizing fasciitis and in their ability to cause soft tissue infections in a mouse model studied. PSMs in S. aureus contribute to the formation of biofilms and detachment of biofilm clusters for dissemination. The presence of the PSMs in MCRF184 and the biofilm genes (Additional file 1: Figure S1), CKU_2420 through CKD_2424 may again enhance the necrotizing fasciitis capability of the strain. PSMs of the $\alpha$-type are known to be cytolytic, and the $\delta$-toxin has been shown to lead to mast cell degranulation. The $\delta$-toxin of MCRF184 (Table 1) is found within the RNAIII gene (CKU_2494) downstream of the agrB gene (CKU_1795). The RNAIII is the effector of the Agr system [6]. An interesting role for the PSM $\alpha 3$ of MCRF184 is their formation of amyloids [37] that are cross- $\alpha$-fibrils, a newly discovered mode of self-assembly characterized by the piling of $\alpha$-helices (Additional file 1: Figure S1C) perpendicular to the fibril axis. Similarly, PSM $\alpha 1$ promotes biofilm stability by preventing disassembly by matrix degrading enzymes and mechanical stress [38].

Table 4 Comparison of vSay of MCRF184 with strain, CA347 identifying SNP differences between them

\begin{tabular}{|c|c|c|c|c|c|c|c|c|}
\hline Nucleotide Position & AA Change & Region & CDS & CDS Position & Change & Codon Change & Polymorphism Type & Protein Effect \\
\hline $1,086,484$ & No & XTP/dITP diphosphatase & $\overline{C K U \_0983}$ & 87 & $\mathrm{~T}>\mathrm{C}$ & TAT > TAC & SNP (transition) & None \\
\hline $1,071,553$ & A & $H P$ & CKU_0988 & 1 & $A>T$ & ATG $>$ TAG & SNP (transversion) & None \\
\hline $1,077,630$ & C & $s s / 12$ & CKU_0998 & 145 & $C>G$ & $A C A>A G A$ & SNP (transversion) & None \\
\hline
\end{tabular}




\section{murl YfcE efb-c fir \\ scn \\ hla \\ ss/12 ss/13 ss/14 $\arg F$

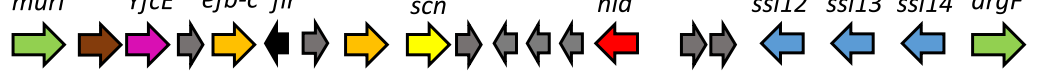

$1,067,411$

$1,081,169$

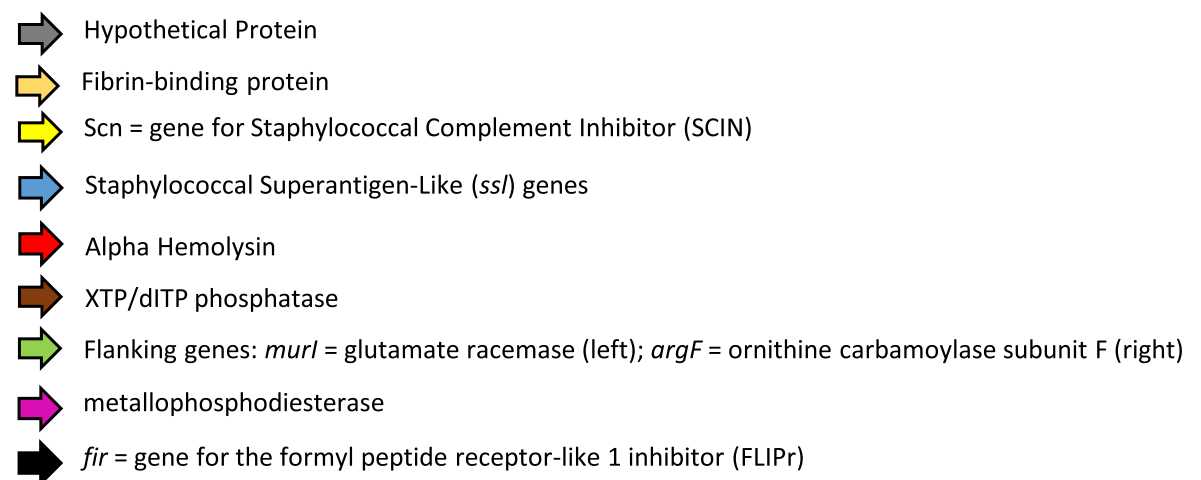

Fig. 4 vSay genomic island. Included in this island is the IEC2 group of genes

\section{Conclusion}

MCRF184's genome contained several distinguishing features, such as a truncated $\nu \mathrm{Sa} \beta$, an incomplete phage and a MGE ${ }^{X Y}$ not seen other $S$. aureus STs. Virulence of this strain likely came from its unique genetic background and SNPs in regulatory elements of virulence genes including $e g c$. It also highlights the fact that there are highly virulent $S$. aureus strains out there which despite lacking the known potent toxins such as Panton-Valentine leukocidin, alpha toxin, etc., are still capable of causing serious, debilitating disease in susceptible individuals.

\section{Methods}

The study was approved by the Marshfield Clinic Research Institute's Institutional Review Board under the study number SHU10105 with waiver of documentation of informed consent.

\section{Bacterial strain}

The $S$. aureus strain MCRF184, was isolated multiple times from a 72-year-old male during the treatment of his necrotizing fasciitis [11], and we sequenced the first isolate's genome.

\section{Genome sequence and comparative analysis}

The MCRF184 genome was sequenced by both a shotgun (single end) and a paired end libraries on a Roche 454 and assembled and annotated as described in Aswani et al 2016 [39] (BioProject PRJNA39571, BioSample SAMN02953006, GenBank CP014791). Its multilocus sequence type (MLST) and lack of SCCmec was deduced from the genome sequence and confirmed by Sanger sequencing and PCR.
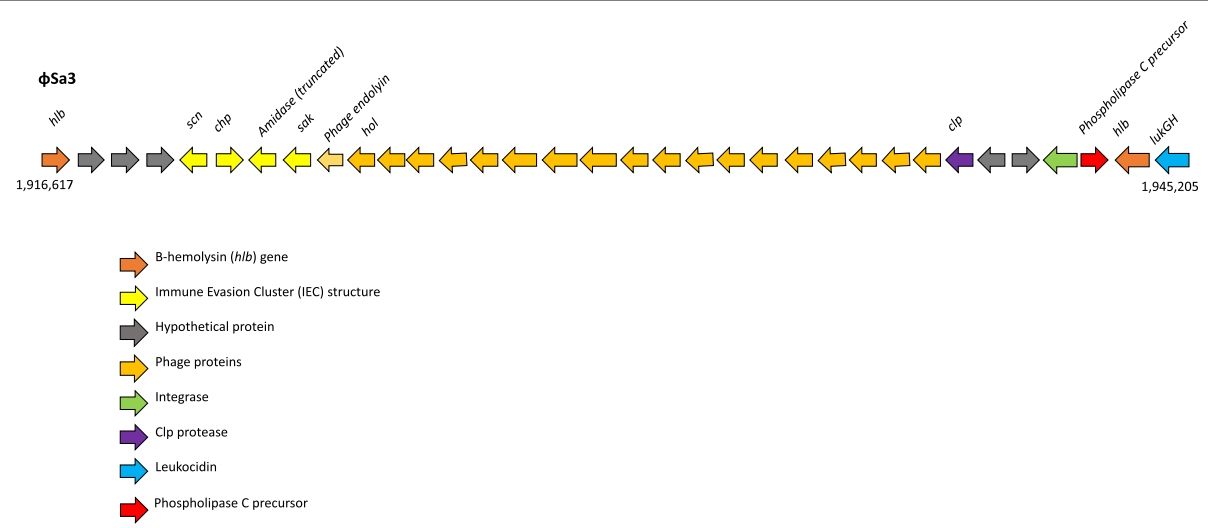

Fig. 5 pSa3 phage-derived region showing genes of the immune evasion cluster (IEC) 

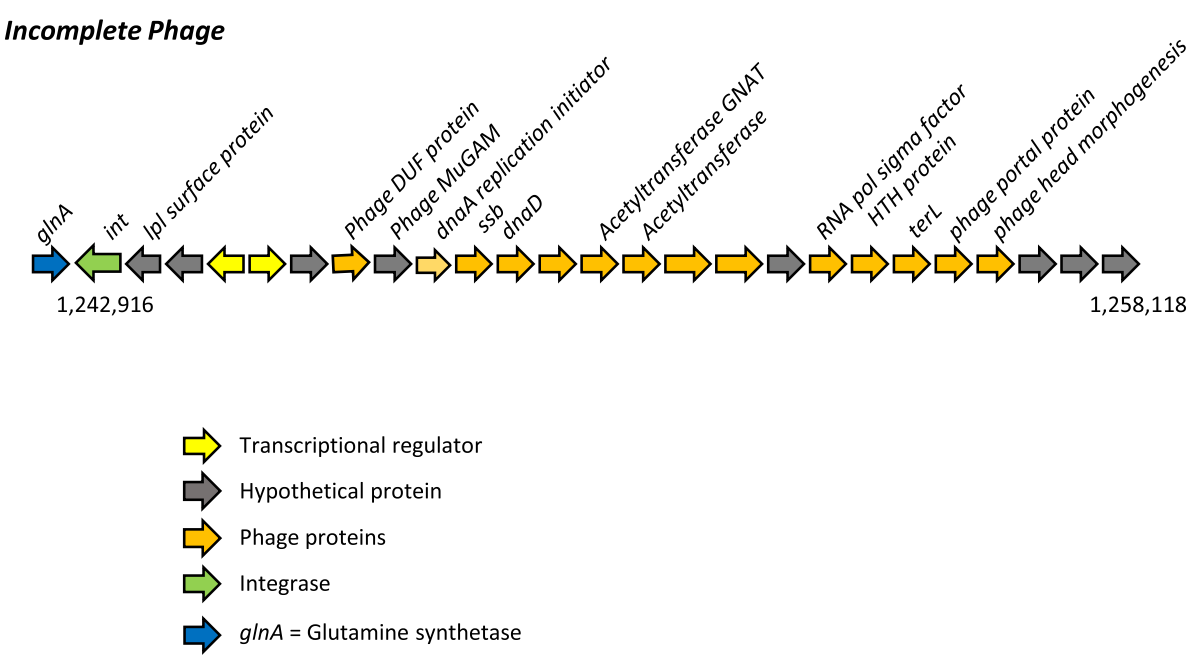

Fig. 6 Incomplete phage showing phage-derived genes

\section{Identification of genomic islands and other putative virulence genes}

Genomic islands in the MCRF815 genome were confirmed using IslandFinder [40] and Zisland explorer [41]. Virulence factors were further identified using VirulenceFinder [42].

\section{Prophage analysis}

PHASTER (PHAge Search Tool - Enhanced Release) was used to analyze prophages in the genome [43]. This program is based on an earlier version called PHAST that detects prophage regions by examination prophage genes and their distance from each other [44].

\section{Single nucleotide polymorphism (SNP) analysis}

SNP Analysis was performed with Geneious 11.0.3 (https://www.geneious.com). To perform the analysis, DNA sequences of the three genomic island, $v \mathrm{Sa} \alpha$, $v \mathrm{Sa} \beta$, and $v$ Say from MCRF184 were aligned with the corresponding island sequences of CA-347 using Geneious Alignment, a global alignment with free end gaps with a $65 \%$ similarity $(5.0 /-4.0)$ cost matrix and gap open penalty of 12 and gap extension penalty of 3. Once aligned, Geneious called variants/SNPs and reported effect of the variants on protein translation using a Bacterial Genetic Code, and merging adjacent variations.

\section{PSM peptide structure modelling}

The predicted protein structure of the $\alpha$-PSMs were determined using SWISS_MODEL Workplace [29] (https:// swissmodel.expasy.org/interactive). The SWISS_MODEL accepted the peptide sequence as input, with no additional parameters required and it generated a PDB file formatted secondary structure, and a descriptive report. The protein structure of the $\beta$-PSMs was modelled using PEP-FOLD3 [27, 28] (http://mobyle.rpbs.univ-paris-diderot.fr/cgi-bin/ portal.py\#forms::PEP-FOLD3). The input was the PSM amino acid sequences to generate a 3-D structure of the peptide using sOPEP (structure Optimized Potential for Efficient structure Prediction) as the model sorter after 100 independent simulations.

MGE $^{X Y}$ (Mobile Genetic Element between orfX and orfY)

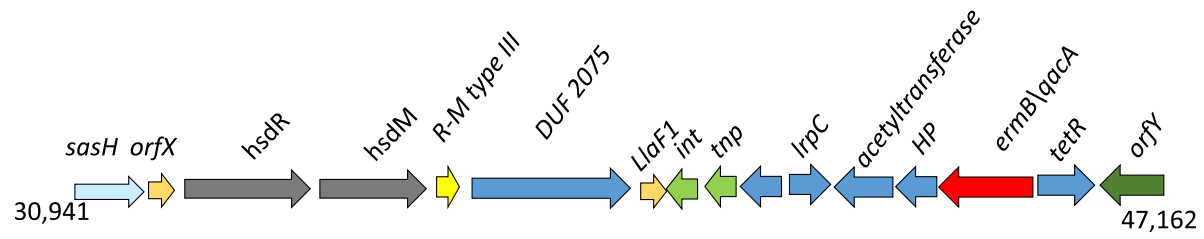

Fig. $7 \mathrm{MGE}{ }^{X Y}$ (Mobile Genetic Element between orfX and orfY) is a region on the genome showing unique mix of antibiotic-resistance genes and mobile genetic elements. Other $\mathrm{S}$. aureus genome regions are shown for comparison. orf $\mathrm{X}$ is also known as $\mathrm{rlmH}$ 


\section{Additional file}

Additional file 1: Figure S1. Phenol soluble modulins (PSM) of S. aureus MCRF184. Panel 1A shows the amino acid sequences of the alpha and beta PSMs arranged from the $\mathrm{N}$-terminus to the $\mathrm{C}$-terminus. Numbers at the right show the net charge of the peptides at $\mathrm{pH} 7.0$, rounded to whole numbers, and considering $\mathrm{N}$-formylation of the initial methionine residue. The highlighted text identifies the amphipathic a-helical domain. Panel 1B shows the location of the genes coding for these PSMs in the genome of MCRF184 core genome. Panel 1C shows the predicted structure of the PSMS using PEP-FOLD (for the alpha PSMs) and SWISS-MODEL Workspace (for the beta PSMs). The residues are color-coded by their position in the peptide chain. Each chain is drawn as a smooth spectrum from blue through green, yellow and orange to red. The $\mathrm{N}$-terminus of the peptides is colored red and the $C$ terminuses are drawn in blue. The structures show the characteristic a-helical structure of the C-terminus ends of the PSMs. (PDF $334 \mathrm{~kb}$ )

\section{Abbreviations}

ICE: integrative conjugative elements; IEC: immune evasion cluster; Ipl: lipoprotein-like; MGE: mobile genetic element; ORFs: open reading frames; PSMs: phenol soluble modulins; PVL: Panton-Valentine leukocidin; SNP: single nucleotide polymorphisms; Ssl: staphylococcal superantigen-like; ST: sequence type

\section{Acknowledgements}

The authors would like to acknowledge Jennifer Kislow and Pravin Kaldhone for their technical assistance. Authors would also like to acknowledge Michigan State University's Core Facility for generating the genome sequence data and initial assembly. Authors are indebted to D. Ashley Robinson from the Department of Microbiology and Immunology, University of Mississippi Medical Center, Jackson, Mississippi, USA who provided expert feedback on several versions of this manuscript. His incisive comments greatly enhanced the completeness of the manuscript.

\section{Funding}

The study was supported, in part, by Marshfield Clinic Research Institute.

Availability of data and materials

The datasets used in the study are available from the NCBI's GenBank under the accession number CP014791.1.

\section{Authors' contributions}

SKS planned and arranged the study. SKS and WRS performed the experiments. VA, MP, FZN, BM, SKS, and WRS analyzed the data. SKS, VA, FZN, and WRS wrote the manuscript with support from all authors. All authors read and approved the final manuscript.

\section{Ethics approval and consent to participate}

Verbal consent from the patient was obtained by the physician to determine the unusual nature of the pathogen's virulence as part of routine clinical care. This study was approved by the Marshfield Clinic Research Institute's Institutional Review Board.

\section{Consent for publication}

$$
\text { Not applicable. }
$$

\section{Competing interests}

The authors declare that they have no competing interests.

\section{Publisher's Note}

Springer Nature remains neutral with regard to jurisdictional claims in published maps and institutional affiliations.

\section{Author details}

${ }^{1}$ Department of Internal Medicine \& Pediatrics, University at Buffalo, Buffalo, New York, USA. ${ }^{2}$ Department of Chemistry \& Biochemistry, University of Oklahoma, Norman, OK, USA. ${ }^{3}$ Center for Human Genetics, 1000 North Oak Avenue \# MLR, Marshfield, WI 54449, USA. ${ }^{4}$ Wisconsin Institute for Discovery,
University of Wisconsin, Madison, WI, USA. ${ }^{5}$ Department of Microbiology, University of Wisconsin -La Crosse, La Crosse, WI, USA.

Received: 26 October 2018 Accepted: 18 December 2018

Published online: 08 February 2019

\section{References}

1. Gordon RJ, Lowy FD. Pathogenesis of methicillin-resistant Staphylococcus aureus infection. Clin Infect Dis. 2008;46(Suppl 5):S350-9.

2. Lindsay JA, Holden MT. Staphylococcus aureus: superbug, super genome? Trends Microbiol. 2004;12(8):378-85.

3. Baba T, Takeuchi F, Kuroda M, Yuzawa H, Aoki K, Oguchi A, et al. Genome and virulence determinants of high virulence community-acquired MRSA. Lancet. 2002;359(9320):1819-27.

4. Diep BA, Gill SR, Chang RF, Phan TH, Chen JH, Davidson MG, et al. Complete genome sequence of USA300, an epidemic clone of community-acquired meticillin-resistant Staphylococcus aureus. Lancet. 2006;367(9512):731-9.

5. Holden MT, Feil EJ, Lindsay JA, Peacock SJ, Day NP, Enright MC, et al. Complete genomes of two clinical Staphylococcus aureus strains: evidence for the rapid evolution of virulence and drug resistance. Proc Natl Acad Sci U S A. 2004;101(26):9786-91.

6. Wang R, Braughton KR, Kretschmer D, Bach TH, Queck SY, Li M, et al. Identification of novel cytolytic peptides as key virulence determinants for community-associated MRSA. Nat Med. 2007;13(12):1510-4.

7. Kennedy AD, Otto M, Braughton KR, Whitney AR, Chen L, Mathema B, et al. Epidemic community-associated methicillin-resistant Staphylococcus aureus: recent clonal expansion and diversification. Proc Natl Acad Sci U S A. 2008; 105(4):1327-32

8. Fitzgerald JR, Holden MT. Genomics of natural populations of Staphylococcus aureus. Annu Rev Microbiol. 2016;70:459-78.

9. Sause WE, Copin R, O'Malley A, Chan R, Morrow BJ, Buckley PT, et al. Staphylococcus aureus strain Newman D2C contains mutations in major regulatory pathways that cripple its pathogenesis. J Bacteriol. 2017.

10. Benson MA, Ohneck EA, Ryan C, Alonzo F 3rd, Smith H, Narechania A, et al. Evolution of hypervirulence by a MRSA clone through acquisition of a transposable element. Mol Microbiol. 2014;93(4):664-81.

11. Morgan WR, Caldwell MD, Brady JM, Stemper ME, Reed KD, Shukla SK. Necrotizing fasciitis due to a methicillin-sensitive Staphylococcus aureus isolate harboring an enterotoxin gene cluster. J Clin Microbiol. 2007:45(2):668-71.

12. Novick RP CG, Penadés JR. The phage-related chromosomal islands of Gram-positive bacteria. Nat Rev Microbiol. 2010; 2010 Aug;8(8):11.

13. Sansevere EA, Robinson DA. Staphylococci on ICE: overlooked agents of horizontal gene transfer. Mob Genet Elements. 2017;7(4):1-10.

14. Dobrindt U, Hochhut B, Hentschel U, Hacker J. Genomic islands in pathogenic and environmental microorganisms. Nat Rev Microbiol. 2004; 2(5):414-24.

15. Stegger M, Driebe EM, Roe C, Lemmer D, Bowers JR, Engelthaler DM, et al. Genome Sequence of Staphylococcus aureus Strain CA-347, a USA600 Methicillin-Resistant Isolate. Genome Announc. 2013;1:4.

16. Martinez-Rubio R, Quiles-Puchalt N, Marti M, Humphrey S, Ram G, Smyth $D$, et al. Phage-inducible islands in the gram-positive cocci. ISME J. 2017:11(4):1029-42.

17. van Wamel WJ, Rooijakkers SH, Ruyken M, van Kessel KP, van Strijp JA. The innate immune modulators staphylococcal complement inhibitor and chemotaxis inhibitory protein of Staphylococcus aureus are located on beta-hemolysin-converting bacteriophages. J Bacteriol. 2006;188(4):1310-5.

18. Madhusoodanan J, Seo KS, Remortel B, Park JY, Hwang SY, Fox LK, et al. An enterotoxin-bearing Pathogenicity Island in Staphylococcus epidermidis. J Bacteriol. 2011;193(8):1854-62.

19. Goerke C, Pantucek R, Holtfreter S, Schulte B, Zink M, Grumann D, et al. Diversity of prophages in dominant Staphylococcus aureus clonal lineages. J Bacteriol. 2009;191(11):3462-8.

20. Novick RP, Christie GE, Penades JR. The phage-related chromosomal islands of gram-positive bacteria. Nat Rev Microbiol. 2010;8(8):541-51.

21. Su J, Liu X, Cui H, Li Y, Chen D, Li Y, Yu G. Rapid and simple detection of methicillin-resistance Staphylococcus aureus by orfX loop-mediated isothermal amplification assay. BMC Biotechnol. 2014;14:8.

22. Noto MJ, Kreiswirth BN, Monk AB, Archer GL. Gene acquisition at the insertion site for SCCmec, the genomic island conferring methicillin resistance in Staphylococcus aureus. J Bacteriol. 2008;190(4):1276-83. 
23. Semmler T, Harrison EM, Lubke-Becker A, Ulrich RG, Wieler LH, Guenther $\mathrm{S}$, et al. A look into the melting pot: the mecC-harboring region is a recombination hot spot in Staphylococcus stepanovicii. PLoS One. 2016; 11(1):e0147150.

24. Costa SS, Viveiros M, Amaral L, Couto I. Multidrug efflux pumps in Staphylococcus aureus: an update. Open Microbiol J. 2013;7:59-71.

25. Amissah NA, Chlebowicz MA, Ablordey A, Tetteh CS, Prah I, van der Werf TS, et al. Virulence potential of Staphylococcus aureus isolates from Buruli ulcer patients. Int J Med Microbiol. 2017;307(4-5):223-32.

26. Cheung GY, Joo HS, Chatterjee SS, Otto M. Phenol-soluble modulins--critical determinants of staphylococcal virulence. FEMS Microbiol Rev. 2014;38(4):698-719.

27. Shen Y, Maupetit J, Derreumaux P, Tuffery P. Improved PEP-FOLD approach for peptide and Miniprotein structure prediction. J Chem Theory Comput. 2014;10(10):4745-58.

28. Thevenet P, Shen Y, Maupetit J, Guyon F, Derreumaux P, Tuffery P. PEPFOLD: an updated de novo structure prediction server for both linear and disulfide bonded cyclic peptides. Nucleic Acids Res. 2012;40:W288-93.

29. Waterhouse A, Bertoni M, Bienert S, Studer G, Tauriello G, Gumienny R, et al. SWISS-MODEL: homology modelling of protein structures and complexes. Nucleic Acids Res. 2018;46(W1):W296-303.

30. Nguyen MT, Kraft B, Yu W, Demircioglu DD, Hertlein T, Burian M, et al. The nuSaalpha Specific Lipoprotein Like Cluster (Ipl) of S. aureus USA300 Contributes to Immune Stimulation and Invasion in Human Cells. PLoS Pathog. 2015;11(6):e1004984.

31. Kotzin BL, Leung DY, Kappler J, Marrack P. Superantigens and their potential role in human disease. Adv Immunol. 1993:54:99-166.

32. Ferry $\mathrm{T}$, Thomas $\mathrm{D}$, Genestier AL, Bes M, Lina G, Vandenesch F, Etienne J. Comparative prevalence of superantigen genes in Staphylococcus aureus isolates causing sepsis with and without septic shock. Clin Infect Dis. 2005; 41(6):771-7.

33. Roetzer A, Gruener CS, Haller G, Beyerly J, Model N, Eibl MM. Enterotoxin Gene Cluster-Encoded SEl and SEIN from Staphylococcus aureus Isolates are Crucial for the Induction of Human Blood Cell Proliferation and Pathogenicity in Rabbits. Toxins (Basel). 2016;8:11.

34. Stach CS, Vu BG, Merriman JA, Herrera A, Cahill MP, Schlievert PM, SalgadoPabon W. Novel tissue level effects of the Staphylococcus aureus enterotoxin gene cluster are essential for infective endocarditis. PLoS One. 2016;11(4):e0154762.

35. Johler S, Giannini P, Jermini M, Hummerjohann J, Baumgartner A, Stephan R. Further evidence for staphylococcal food poisoning outbreaks caused by egc-encoded enterotoxins. Toxins (Basel). 2015;7(3):997-1004.

36. Cuthbertson L, Nodwell JR. The TetR family of regulators. Microbiol Mol Biol Rev. 2013;77(3):440-75.

37. Salinas N, Colletier JP, Moshe A, Landau M. Extreme amyloid polymorphism in Staphylococcus aureus virulent PSMalpha peptides. Nat Commun. 2018;9(1):3512.

38. Schwartz K, Syed AK, Stephenson RE, Rickard AH, Boles BR. Functional amyloids composed of phenol soluble modulins stabilize Staphylococcus aureus biofilms. PLoS Pathog. 2012;8(6):e1002744.

39. Aswani V, Mau B, Shukla SK. Complete Genome Sequence of Staphylococcus aureus MCRF184, a Necrotizing Fasciitis-Causing Methicillin-Sensitive Sequence Type 45 Staphylococcus Strain. Genome Announc. 2016;4:3.

40. Dhillon BK, Laird MR, Shay JA, Winsor GL, Lo R, Nizam F, et al. IslandViewer 3: more flexible, interactive genomic island discovery, visualization and analysis. Nucleic Acids Res. 2015;43(W1):W104-8.

41. Wei W, Gao F, Du MZ, Hua HL, Wang J, Guo FB. Zisland explorer: detect genomic islands by combining homogeneity and heterogeneity properties. Brief Bioinform. 2017;18(3):357-66

42. Joensen KG, Scheutz F, Lund O, Hasman H, Kaas RS, Nielsen EM, Aarestrup FM. Real-time whole-genome sequencing for routine typing, surveillance, and outbreak detection of verotoxigenic Escherichia coli. J Clin Microbiol. 2014;52(5):1501-10.

43. Arndt D, Grant JR, Marcu A, Sajed T, Pon A, Liang Y, Wishart DS. PHASTER: a better, faster version of the PHAST phage search tool. Nucleic Acids Res. 2016:44(W1):W16-21

44. Zhou Y, Liang Y, Lynch KH, Dennis JJ, Wishart DS. PHAST: a fast phage search tool. Nucleic Acids Res. 2011:39:W347-52

\section{Ready to submit your research? Choose BMC and benefit from:}

- fast, convenient online submission

- thorough peer review by experienced researchers in your field

- rapid publication on acceptance

- support for research data, including large and complex data types

- gold Open Access which fosters wider collaboration and increased citations

- maximum visibility for your research: over $100 \mathrm{M}$ website views per year

At $\mathrm{BMC}$, research is always in progress.

Learn more biomedcentral.com/submissions 